\title{
La classe inversée en mode virtuel : portrait d'une formule gagnante
}

Auteures

Suzie Tardif, doctorante en éducation, Université du Québec à Chicoutimi, Canada,

suzie.tardif@uqac.ca

Marilyne Rivard, enseignante en mathématiques, cégep de Saint-Félicien, Canada,

mrivard@cegepstfe.ca 


\section{REVUE HYBRIDE DE L’ÉDUCATION}

\section{Résumé}

Cet article présente une initiative pédagogique mise en place lors de la pandémie de COVID-19 par une enseignante en mathématiques du cégep de Saint-Félicien (Saguenay-Lac-Saint-Jean). Adepte de la classe inversée, elle a décidé d'exploiter ce dispositif pédagogique en mode entièrement virtuel. Elle considère avoir réussi à maintenir la motivation de ses étudiants en créant, entre autres, des capsules vidéo interactives et des activités d'apprentissage à réaliser à la suite de leur visionnement. Dans cet article, la mise en œuvre et les retombées perçues par l'enseignante de cette expérience seront notamment décrites.

Mots-clés : classe inversée; apprentissage actif; capsules interactives; enseignement des mathématiques; enseignement collégial 


\section{$\&$}

\section{REVUE HYBRIDE DE L'ÉDUCATION}

\section{Mise en contexte}

La pandémie de COVID-19 a amené les enseignants de tous les ordres d'enseignement à repenser leurs formules pédagogiques, notamment parce qu'ils devaient enseigner à distance. Ce constat est marqué au postsecondaire puisque les enseignants ont dû prioriser l'enseignement virtuel pendant plusieurs mois. Des initiatives innovantes ont dès lors été mises en place par plusieurs d'entre eux afin de maintenir la motivation et l'engagement de leurs étudiants.

C'est notamment le cas d'une enseignante en mathématiques du cégep de Saint-Félicien (Saguenay-Lac-Saint-Jean) qui a rapidement dû revoir ses façons de faire au printemps 2020. Lors de la première vague de COVID-19, elle avoue avoir vécu beaucoup d'incertitudes liées à l'arrêt obligatoire de toutes ses activités d'enseignement. Lorsqu'elle a appris que les cours qu'elle proposait devaient obligatoirement être pilotés à distance, elle a rapidement modifié la façon dont elle enseignait. Elle ne souhaitait pas réaliser ses cours en mode synchrone considérant, entre autres, les enjeux technologiques qui pouvaient freiner son enseignement (difficulté de connexion pour les étudiants, enjeux liés à la démonstration des calculs pour réaliser un problème mathématique, etc.).

Conséquemment, elle a décidé d'exploiter la stratégie pédagogique de classe inversée, pour laquelle elle avait déjà développé un intérêt. Elle s'est dès lors inspirée de l'expérience de deux de ses collègues qui avaient utilisé cette approche pédagogique bien avant la pandémie pour la mettre en œuvre.

Autodidacte, cette enseignante indique ne pas avoir suivi de formation liée à cette approche, mais en avoir appris les fondements dans le cadre de ses études supérieures spécialisées (DESS) en enseignement au collégial de l'Université de Sherbrooke. Elle précise également que le colloque annuel de l'Association québécoise de pédagogie collégiale (AQPC) demeure une occasion unique de susciter son intérêt envers différentes approches pédagogiques d'apprentissage actif (Chi et Wylie, 2014 ; Hake, 1998) grâce aux ateliers qui y sont présentés, lesquelles ont été exploitées dans sa mise à l'essai de la classe inversée. Par définition, la classe inversée se résume avec l'idée que :

Les activités hors de la classe sont axées sur l'appropriation autonome de contenus médiatisés, ce qui rend une partie de l'expertise disponible dans un espace virtuel, accessible en tout temps à l'étudiant. Les activités en classe se réalisent tant qu'à elles par la valorisation de la pédagogie active, selon des modalités variées (Nizet, Baliano et Meyer, 2016, p.41). 


\section{REVUE HYBRIDE DE L'ÉDUCATION}

Comme le synthétisent Lakrami et ses collaborateurs (2018), cette stratégie sous-entend que les étudiants se présentent en classe pour réaliser des activités d'apprentissage de manière active, souvent réalisées en groupe, liées au visionnement de capsules portant sur les contenus abordés.

Or, comment fait-on pour utiliser cette approche lorsqu'il nous est interdit de réunir ses étudiants en classe ? Une entrevue a ainsi été réalisée avec cette enseignante de mathématiques qui semble avoir trouvé une formule efficace pour remédier à cette contrainte présentielle. Les lignes qui suivent brossent un portrait du scénario pédagogique qu'elle a conçu à la suite des contraintes sanitaires imposées par la pandémie de la COVID19.

\section{Déroulement : Une combinaison gagnante de formules pédagogiques}

D'entrée de jeu, cette enseignante précise que l'utilisation exclusive de capsules vidéo ne saurait être efficace pour permettre à ses étudiants de développer les compétences visées en mathématiques dans ses cours. Pour elle, le succès lié à l'utilisation de la classe inversée découle de l'exploitation de plusieurs formules pédagogiques : «Seules, les capsules ne suffisent pas. Si tu ne peux pas tâter le pouls de tes étudiants, ça ne sert à rien ". Dans cet ordre d'idées, la figure 1 présente le scénario pédagogique qu'elle a envisagé.

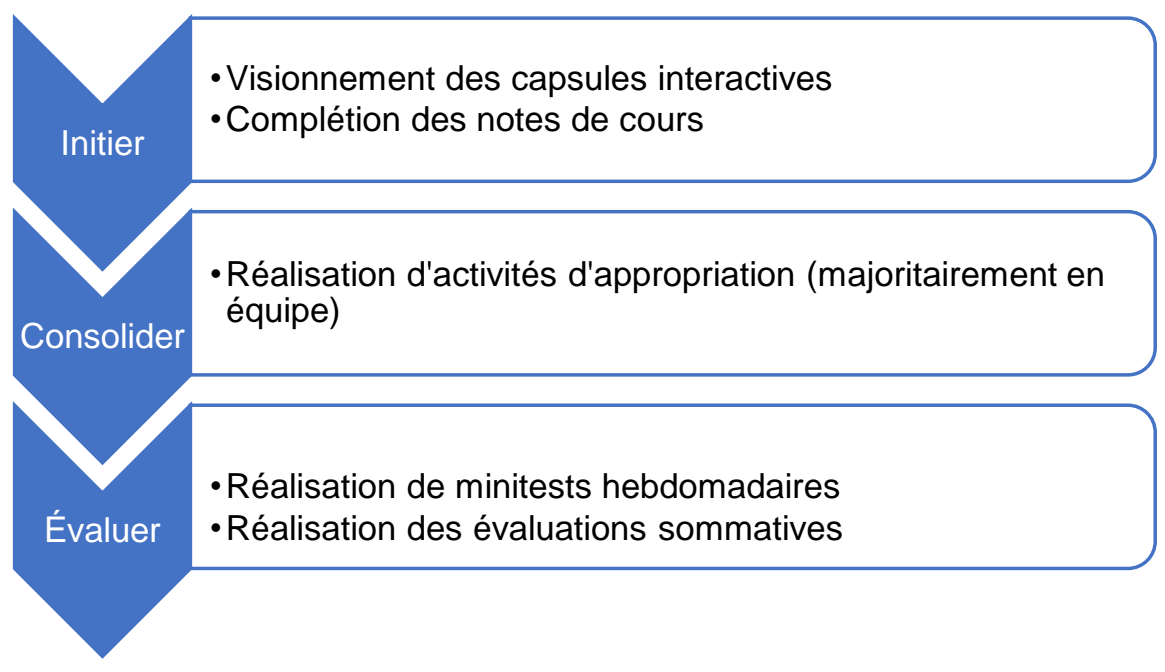

Figure 1 : Scénario pédagogique d'une classe inversée en mode virtuel 


\section{8}

\section{REVUE HYBRIDE DE L'ÉDUCATION}

\section{Initier les apprentissages}

Chaque semaine, les étudiants doivent d'abord visionner une capsule, disponible sur la plateforme Moodle, d'une durée de 5 à 30 minutes, portant sur un concept en mathématiques. Ces capsules sont dites interactives puisque des questions sur les contenus abordés sont posées aux étudiants lorsqu'ils les visionnent. Ceux-ci doivent également compléter les notes de cours liées au concept abordé dans la capsule, lesquelles sont disponibles en format numérique et modifiables au besoin.

Pour réaliser les capsules, différentes applications peuvent être utilisées (ex.: Panopto). L'enseignante consultée privilégie deux applications qui lui plaisent davantage et avec lesquelles elle se sent plus à l'aise.

Plus précisément, elle utilise Explain everything pour réaliser une première version de la capsule. Cette application demeure particulièrement intéressante en mathématiques puisqu'elle permet la réalisation de tutoriels via un tableau blanc interactif et collaboratif. En utilisant un iPad, il est dès lors possible d'enregistrer une capsule dans laquelle un problème mathématique est résolu. Les étudiants peuvent voir la démarche, les calculs et les explications qui s'y rapportent.

Par la suite, l'enseignante utilise $H 5 P$, un logiciel libre de création de contenu interactif disponible sur Moodle, pour rendre interactive la capsule crée. En effet, H5P permet d'ajouter du texte, des images ou des questions dans la première version réalisée de la capsule. L'étudiant peut ainsi être amené à répondre, obligatoirement ou non, à certaines questions de toutes formes (ex. : vrai ou faux, exercices d'association, choix de réponses ou à développement) sur le contenu présenté avant de poursuivre son visionnement. Le seul inconvénient de cette application demeure qu'il n'est actuellement pas possible pour l'enseignant de consulter les réponses attribuées aux questions par les étudiants. De leur côté, les étudiants ont accès aux corrigés de toutes les questions posées.

\section{Consolider les apprentissages}

Après avoir visionné les capsules, les étudiants sont invités à réaliser des activités d'apprentissage liées au contenu abordé dans celles-ci lors des périodes de cours. Contrairement à ce qu'elle réalisait antérieurement, les activités proposées par cette enseignante ne sont plus exclusivement des résolutions de problèmes à réaliser individuellement. En effet, elle voulait prioriser un apprentissage actif par ses étudiants. Tel que synthétisé par Normand (2017), 


\title{
$\&$
}

\section{REVUE HYBRIDE DE L'ÉDUCATION}

\begin{abstract}
L'apprentissage actif comporte des méthodes pédagogiques efficaces qui nécessitent de la part de l'étudiant un traitement en profondeur de l'information. Le professeur qui en tient compte proposera aux étudiants des activités qui impliquent des modes d'engagement actif, constructif et même mieux, interactif (p. 11).
\end{abstract}

Durant la pandémie, elle a ainsi proposé des exercices qui permettent de comprendre davantage les concepts abordés (ex.: construction de schémas conceptuels, associations de définitions, texte troué à compléter avec une banque de mots) avant de les appliquer à travers une résolution de problèmes. Pour cette enseignante, ces activités demeurent particulièrement intéressantes pour les étudiants d'un point de vue pédagogique : "Ces exercices, ça amène leur compréhension plus loin, ils réfléchissent autrement, ce n'est pas nécessairement un calcul ».

Pour cette étape du scénario pédagogique, l'enseignante privilégie le travail d'équipe considérant toutes les retombées pédagogiques qui en découlent. Pour ce faire, elle conçoit des équipes de 3 ou 4 étudiants sur la plateforme Teams. Elle apprécie cette plateforme collaborative puisqu'elle est en mesure de visualiser les travaux de ses étudiants et d'intervenir au besoin en ajoutant des commentaires.

Les étudiants n'ont pas accès aux corrigés des activités proposées. Ils doivent s'entraider pour les réaliser et prioriser le soutien de leurs confrères lorsqu'ils rencontrent des difficultés. Ils doivent cependant aviser l'enseignante lorsqu'ils ont terminé celles-ci pour qu'elle puisse réagir à leurs réponses et offrir des explications supplémentaires au besoin. Cet échange lui permet d'outiller davantage les étudiants qui présentent des difficultés, mais également de brosser un portrait des apprentissages acquis par ses étudiants. Selon l'enseignante consultée, le fait de travailler avec ses pairs présente de nombreux avantages au point de vue pédagogique. Le tout permet aux étudiants de vulgariser les concepts abordés, mais aussi de consolider leurs apprentissages, notamment lorsqu'un collègue partage sa compréhension de l'activité présentée : «Les différentes perceptions des étudiants créent des échanges super instructifs qui amènent les étudiants à se questionner davantage sur leur compréhension du sujet ».

\section{Évaluer les apprentissages}

Deux types d'évaluation sont ensuite proposés aux étudiants. Dans un premier temps, une évaluation à visée formative, sous forme de minitests, doit être complétée hebdomadairement et individuellement par les étudiants, après avoir réalisé les activités d'apprentissage liées au contenu d'une capsule. Ces minitests permettent de consolider les apprentissages réalisés par les étudiants et d'évaluer leur degré de 


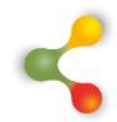

\section{REVUE HYBRIDE DE L'ÉDUCATION}

compréhension du concept abordé. L'enseignante accorde $100 \%$ à tous ceux qui obtiennent plus de 6 sur 10 à cette forme d'évaluation. Pour elle, l'attribution de ce résultat demeure une forme de motivation extrinsèque par régulation externe (Deci et Ryan, 2000) qui est efficace auprès de ses étudiants : «Mon objectif c'est de voir s'ils ont compris l'essentiel. Eux, ça leur donne un sentiment réconfortant qu'ils comprennent et ils vont se chercher des points. II faut un nanane [une récompense] pour que les paresseux le fassent aussi. 10 minitests qui valent pour $10 \%$ de la note finale, ça les motive ».

Seules les deux évaluations sommatives ont lieu en classe, afin de pouvoir corriger plus facilement les traces des démarches des étudiants et éviter toute forme de plagiat. Évidemment, cette façon de faire pourrait être modifiée si la présence en classe des étudiants n'est pas permise par la santé publique. Cette enseignante s'est d'ailleurs tournée vers son conseiller pédagogique lors de la première vague de COVID-19 pour connaitre les options possibles sur Moodle en ce qui a trait aux évaluations en ligne, puisque la présence en classe de ses étudiants était alors interdite.

\section{Apports et prospectives}

\section{Les apports}

Dans un premier temps, selon cette passionnée des mathématiques, les apports perçus se rapportent à la qualité de l'enseignement. En effet, elle considère que ses capsules interactives permettent aux étudiants d'avoir accès à du matériel pédagogique de grande qualité, bien vulgarisé, qu'ils peuvent visionner à plusieurs reprises au besoin : « À mon avis, les capsules permettent de transmettre plus efficacement mon contenu. II n'y a pas d'erreur, tout est bien présenté. [...] lls peuvent réécouter des segments lorsqu'ils ont moins bien compris quelque chose ». Elle avoue, avec humour, qu'elle doit toutefois réaliser plusieurs enregistrements avant de considérer sa capsule comme étant parfaite.

Dans le même ordre d'idées, elle apprécie les changements apportés dans sa pratique tout au long de la pandémie. Considérant que les plateformes numériques qu'elle connaissait ne permettaient pas aux étudiants de réaliser facilement des résolutions de problème, elle a dû penser rapidement à une alternative. Elle a donc conçu des activités d'apprentissage qui se veulent être différentes de ce qu'elle proposait antérieurement. Elle les apprécie particulièrement puisqu'elles facilitent, à son avis, la compréhension de ses étudiants : "Avant, je me concentrais plus sur s'ils étaient ou non capables de faire un calcul. Là, je regarde s'ils comprennent ce qu'ils doivent faire et pourquoi. J'ai amené mes questions ailleurs et je m'assure qu'ils comprennent maintenant bien le concept avant de l'appliquer ». 


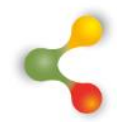

\section{REVUE HYBRIDE DE L'ÉDUCATION}

Interrogés sur leur appréciation du cours, les étudiants de cette enseignante ont rapporté plusieurs points positifs à cette méthode d'enseignement, notamment qu'ils se sentent moins seuls et qu'ils apprécient l'interactivité découlant des capsules: " Le pouls de mes étudiants est vraiment bon, ils m'ont dit qu'ils avaient l'impression que j'étais beaucoup plus présente, qu'ils n'étaient pas seuls à l'autre bout de l'écran et que je me souciais de leur compréhension avec les capsules, que c'était comme si j'étais avec eux en classe et que je leur posais des questions.» Ils sont d'avis que la classe inversée a nécessité une plus grande autonomie de leur part, cette situation étant souvent relevée par l'enseignement à distance (Nizet, Galiano et Meyer, 2016; Younès, Caira, Ionascu et Cracium, 2016; Zorn, Feffer, Bauer et Dillenseger, 2020). Cette plus grande autonomie se conjugue toutefois avec un plus grand sentiment de contrôle envers leurs apprentissages (Zorn, Feffer, Bauer et Dillenseger, 2020).

Enfin, les conseillers pédagogiques de son collège sont demeurés des alliés importants de cette enseignante lors de cette mise à l'essai. En effet, ils se sont montrés constamment disponibles lorsqu'elle avait des questions sur les applications exploitées, ce qui lui a permis d'être rapidement à l'aise avec celles-ci : « Je pouvais poser ma question et avoir une réponse rapidement, sans avoir à visionner des capsules explicatives sur les applications que j'utilisais ». Une retombée intéressante de cet accompagnement demeure que des capsules explicatives ont été réalisées par les conseillers pédagogiques consultés en fonction des besoins et questionnements relevés par cette enseignante: " pour les questions que j'avais sur H5P, ma conseillère pédagogique responsable des TIC a fait des capsules là-dessus pour aider d'autres enseignants intéressés. Ça a permis aux conseillers pédagogiques de mieux cibler leurs interventions par la suite ».

\section{Les défis rencontrés}

L'application de la classe inversée en mode virtuel ne s'est évidemment pas réalisée sans heurts. Un premier défi rencontré par cette enseignante demeure le temps à consacrer à l'appropriation des applications (ex. : H5P) et à la réalisation des capsules : "Je mets plus de temps que j'en mettais avant à préparer mes cours, c'est une évidence ! ». Toutefois, cette enseignante accueille positivement cet investissement puisqu'elle est grandement satisfaite des retombées qui en découlent.

Qui plus est, cette enseignante est consciente qu'elle ne pourra guère utiliser de nouveau toutes les capsules qu'elle a réalisées. En effet, considérant qu'elles demeurent constamment personnalisées aux besoins et au cheminement de ses étudiants (ex. : donner des explications supplémentaires sur les difficultés relevées dans une évaluation), elle est d'avis qu'il ne serait pas pertinent de les proposer à ses futurs étudiants. 


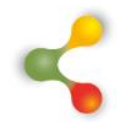

\section{REVUE HYBRIDE DE L'ÉDUCATION}

En contrepartie, selon elle, cette adaptation demeure un facteur de succès de ses capsules puisque ses étudiants se sentent grandement concernés par ses propos. Conséquemment, elle envisage de réaliser certaines capsules intemporelles portant sur divers concepts abordés dans ses cours, mais privilégiera la réalisation de nouvelles capsules pour chacun d'entre eux.

Dans un autre ordre d'idées, elle déplore le manque de contacts avec ses étudiants et de spontanéité occasionnés par l'enseignement à distance. Elle est toutefois d'avis qu'il est tout de même possible de créer un lien avec eux. Pour ce faire, elle s'assure de réaliser des suivis réguliers avec ses étudiants par l'entremise de la zone de clavardage proposée sur Teams. À mesure que la session avance, les étudiants sont de plus en plus à l'aise de lui écrire, avoue-t-elle : "Le lien finit par se créer, certains de mes étudiants m'écrivent ou m'appellent vraiment souvent». Elle communique également avec eux de manière régulière sur un groupe privé Facebook en partageant des nouvelles concernant les cours (ex. : préciser que les résultats des évaluations sont disponibles).

\section{Conclusion}

Cette expérience de classe inversée occasionnée par les contraintes liées à la pandémie a permis à une enseignante de bonifier ses pratiques pédagogiques. Lorsque la situation sanitaire sera résolue, elle envisage de modifier ses façons d'enseigner en formule présentielle compte tenu des apprentissages découlant de cette expérience : "Ma pratique va tellement changer quand on va revenir à la normal ». Concrètement, elle désire enseigner en mode inversé, en exploitant le temps en classe pour permettre à ses étudiants de réaliser divers exercices. Elle privilégiera plus de formules pédagogiques issues de l'apprentissage actif qui favorisent les interactions entre les étudiants telles que les études de cas ou les plénières (Nizet et al., 2016).

Lors de l'entrevue, cette enseignante a précisé d'entrée de jeu qu'elle tentait constamment de s'adapter aux besoins de ses étudiants : "L'étudiant est au centre de mes préoccupations, j'essaie toujours de m'adapter à leur réalité, tout ça en faisant en sorte de faire de mon mieux pour que les concepts passent bien ». Ce constat semble être on ne peut plus véridique à la lumière de cette expérience découlant des changements imposés par la pandémie de la COVID-19. Sa créativité et sa proactivité ont su être mises en lumière à travers les changements apportés à sa pédagogie et pourront certainement être une source d'inspiration pour d'autres enseignants. 


\section{REVUE HYBRIDE DE L'ÉDUCATION}

\section{Références}

Chi, M.T.H. et Wylie, R. (2014). The ICAP Framework: Linking Cognitive Engagement to Active Learning Outcome. Educational Psychologist, 49(4), 219-243.

Deci, E. L. et Ryan, R. M. (2000). Self-Determination Theory and the Facilitation of Intrinsic Motivation, Social Development, and WellBeing. American Psychologist, 55(1), 68-78. https://selfdeterminationtheory.org/SDT/documents/2000 RyanDeci SDT.pdf

Hake, R. R. (1998). Interactive-engagement versus Traditional Methods: A Six-thousandstudent Survey of Mechanics Test Data for Introductory Physics Courses. American Journal of Physics, 66(1), 64-74.

Lakrami, F., Labouidya, O. et Elkamoun, N. (2018). Pédagogie universitaire et classe inversée : vers un apprentissage fructueux en travaux pratiques. Revue Internationale de Pédagogie de l'Enseignement Supérieur, 34(3). DOI : https://doi.org/10.4000/ripes.1793

Nizet, I., Galiano, O. et Meyer, F. (2016). Vers un cadrage théorique pour comprendre la classe inversée. Dans A. Dumont et D. Berthiaume (dir.), La pédagogie inversée (p. 39-50). De Boeck Supérieur.

Normand, L. (2017). L'apprentissage actif: une question de risques...calculés. Pédagogie collégiale, 31(1), 5-12, en ligne: https://eduq.info/xmlui/bitstream/handle/11515/37485/normand-311-2017.pdf?sequence $=2$

Université de Sherbrooke (2011). Le SSF veille. Faire la classe mais à l'envers : la flipped classroom. Université de Sherbrooke. https://www.usherbrooke.ca/ssf/veille/perspectives-ssf/numerosprecedents/novembre-2011/le-ssf-veille/faire-la-classe-mais-alenvers-la-flipped-classroom.

Younès, N., Caira, F., lonascu, I. et Cracium, D. (2016). Éléments pédagogiques d'une classe inversée. Dans $A$. Dumont et $D$. Berthiaume (dir.), La pédagogie inversée (p.135-147). De Boeck Supérieur.

Zorn, C., Feffer, M.-L., Bauer, É. et Dillenseger, J.-P. (2020). Évaluation d'un dispositif de continuité pédagogique à distance mis en place auprès d'étudiants MERM pendant le confinement sanitaire lié au COVID-19. Journal of Medical Imaging and Radiation Sciences. https://doi.org/10.1016/j.jmir.2020.08.020 